\title{
FATORES PSICOLÓGICOS ASSOCIADOS À FALTA DE MEMÓRIA EM IDOSOS:
}

programa Zen Sênior como recurso terapêutico - revisão narrativa e análise descritiva

PSYCHOLOGIAL FACTORS RELATED TO MEMORY LOSS IN ENDERLIES: Zen

Sênior program as a therapeutic resource - narrative revision and descriptive analysis

\section{Juliana Morgana Stedile}

Psicóloga de Atenção Integral à Saúde, especializada em Neuropsicologia, atua na Unimed Litoral com grupos de promoção à saúde.

\section{Parluci Camila Gomes}

Psicóloga de Atenção Integral à Saúde, especializada em Neuropsicologia, atua na Unimed Litoral com grupos de promoção à saúde.

\section{Tamiris Nunes Markoski}

Nutricionista de Atenção Integral à Saúde, Mestre em gerontologia UFSM-RS, atua na Unimed Litoral com grupos de promoção à saúde 


\title{
RESUMO
}

O envelhecimento pode provocar alterações cognitivas em idosos, especialmente na memória. O objetivo deste estudo foi identificar os principais fatores psicológicos associados à falta de memória em idosos através de artigos publicados nos últimos dez anos disponibilizados nas principais plataformas de pesquisa do país e relatar as experiências vivenciadas com o programa Zen Sênior, voltado ao gerenciamento de estresse e ansiedade desta faixa etária. Nos artigos selecionados foram identificados ansiedade, estresse, depressão, autoeficácia e metamemória como os principais fatores psicológicos relacionados à falta de memória em idosos, sendo estes o foco principal quando este tema é investigado. Em seguida, foram apontados os fatores socioeconômicos e clínicos. Entretanto, as pesquisas não apontaram dados significativos para respaldar os efeitos destes no uso na memória. Face a esta dubiedade de fatores, torna-se essencial que as práticas de saúde voltadas ao idoso atuem na interdisciplinaridade focando essencialmente na saúde mental. Para tal, o programa Zen Sênior da Unimed Litoral contribui neste aspecto com o intuito de amenizar os impactos dos fatores psicológicos no desempenho da memória dos idosos.

Palavras-chave: Fatores psicológicos. Queixas de memória. Dificuldade de memória. Idosos.

\begin{abstract}
Aging may cause cognitive disorders in the elderly, especially in memory. The objective of this study was to identify key psychological factors associated with memory loss in the elderly through articles published in recent ten years available in the main research platforms in the country and report the experiments from Zen Sênior program, which objective was stress and anxiety management. In the selected articles the main psychological factors related to memory loss was anxiety, stress, depression, self-efficacy and metamemory, these being the main focus when this topic is investigated. Then, it was appointed the socioeconomic and clinical factors. However, the research showed no significant data to support the effects of these in memory. Due the dubiousness of these factors, it is essential that health practices aimed at the elderly act on interdisciplinarity, focusing primarily on mental health. To this end, the program Zen Sênior from Unimed Litoral contributes to soften the impact of psychological factors on the memory performance of the elderly.
\end{abstract}

Keywords: Psychological factors. Complaints of memory. Memory difficulties. Elderly. 


\section{INTRODUÇÃO}

A Organização Mundial de Saúde (OMS) estabeleceu em 2010 a idade de 65 anos como início da senescência para os países desenvolvidos e 60 anos para os países em desenvolvimento. $\mathrm{O}$ crescimento da população idosa brasileira representou mais que o dobro do crescimento da população total no ano 2008. Estima-se que a média de vida do brasileiro chegará a 81 anos em 2050, apontando que o país se encaminha para um perfil de população cada vez mais idosa. Apesar de ser uma conquista relevante, que resulta da crescente atenção dada ao idoso, este avanço traz consigo, ao mesmo tempo, o desafio de descobrir como este estágio de vida pode ser melhor usufruído a nível individual, social e psicológico (FERREIRA \& BARHAN, 2011).

No decurso do envelhecimento, os idosos apresentam maior vulnerabilidade a doenças e uma parcela significativa desta população é acometida por alterações cognitivas. Para Carvalho, Neri \& Yassuda (2010) o envelhecer implica na redução da velocidade de processamento da informação, que afeta o desempenho cognitivo, especialmente atenção, memória operacional e memória declarativa. Estas dificuldades refletem um descontentamento em relação às habilidades do sujeito e podem causar diversos prejuízos, especialmente na esfera social, visto que está presente em quase todas as atividades desenvolvidas no cotidiano.

No decorrer das duas últimas décadas, foram elaboradas diversas hipóteses com o objetivo de explicar este declínio no desempenho de idosos em tarefas de memória. Para Yassuda et al. (2005), a primeira hipótese, a do desuso, indica que o estilo de vida do idoso pode não favorecer o funcionamento integral da memória, devido a aposentadorias, mudanças de papeis sociais e afastamento da estimulação necessária. A outra hipótese indica o envelhecimento biológico cerebral, que pode ser responsável pela lentificação, causada pela perda de neurônios ou a redução de conexão entre eles. Ainda, para os autores algumas hipóteses sugerem que o estilo cognitivo do idoso seja menos eficiente por falta de estimulação adequada.

Estas mudanças neuropsicológicas, principalmente os déficits cognitivos, podem se relacionar a diversos fatores, dentre eles, sintomas demenciais. A demência é uma síndrome caracterizada especialmente pelo declínio da memória associado ao déficit de pelo menos uma outra função cognitiva (linguagem, agnosias, apraxias, funções executivas) com intensidade capaz de interferir na funcionalidade e no desempenho social (APA, 2002). Resultados de uma pesquisa que envolveu avaliações neuropsicológicas de indivíduos com demência, apontam que 
o desempenho cognitivo cai rapidamente em todas as áreas (CHARCHAT-FIHMAN et al., 2005).

As queixas de dificuldade de memória podem indicar de fato uma dificuldade cognitiva, entretanto, estudos sugerem que as queixas estão principalmente associadas a fatores psicológicos, como ansiedade, depressão, alta exigência pessoal, metamemória, além dos níveis de escolaridade (PAULO \& YASSUDA, 2009). Outros pesquisas corroboram a ideia de que transtornos psicológicos como, por exemplo, o Transtorno Depressivo Maior, podem vir acompanhados de prejuízos cognitivos, e vice-versa, e que as queixas de memória podem também estar relacionadas à traços de personalidade, estados emocionais e estresse, sendo este último, de natureza comportamental, hormonal e autoperceptiva (SANTOS et al., 2012). Para Huf, Lopes \& Rozenfeld (2000), as dificuldades de memória podem também ser associados a reações adversas a grupos farmacológicos, com destaque os benzodiazepínicos que além do risco de dependência, podem gerar prejuízos cognitivos, mesmo após a interrupção do medicamento. Ainda, as falhas na memória podem estar associadas aos distúrbios do sono (MULLER \& GUIMARÃES, 2007), desta forma, manter-se ativo através da prática regular de atividade física, reflete na autonomia e na obtenção da qualidade de vida, vindo a interferir de maneira positiva para minimizar os declínios de memória declarativa (CODEIRO et al., 2014).

Frente a esta situação, a realização de programas neuropsicológicos para idosos, com intervenções holísticas integradas pelas áreas cognitiva, emocional e social, contribuem para a prevenção de declínios cognitivos, como sistemas de memória, promovendo saúde mental, maior autonomia e independência funcional. Ainda, para Amodeo, Maria Netto \& Fonseca (2010), estas atividades realizadas no contexto grupal favorecem maior motivação e integração social para seus participantes.

Devido ao impacto das queixas de memória nas pessoas idosas, o presente artigo busca apresentar os principais fatores psicológicos associados à falta de memória em idosos, e apresentar como uma possibilidade de recurso terapêutico o programa Zen Sênior de Gerenciamento de Estresse e Ansiedade que vem sendo realizado na Unimed Litoral, para idosos com médio e alto nível de estresse e ansiedade e queixas de falta de memória.

O primeiro e segundo capítulo deste artigo apresenta um estudo diacrônico sobre os principais fatores psicológicos, socioeconômicos e clínicos que influenciam na queixa de memória em idosos. O terceiro capítulo apresenta o relato de experiência do programa Zen Sênior com recurso terapêutico para esta demanda. 


\section{METODOLOGIA}

A busca pelos referenciais teóricos ocorreu em bases de dados eletrônicas como SciELO Brasil e Lilacs e foram selecionados 12 artigos de natureza empírica e de revisão bibliográfica. A análise considerou os objetivos dos estudos, amostras e principais fatores associados à queixa de memória em idosos. A partir da leitura destas publicações selecionadas, foi identificado que os fatores psicológicos são os mais evidentes e presentes nas queixas de falta de memória em idosos. Desta forma, o programa Zen Sênior será apresentado como um relato de experiência de recurso terapêutico para esta demanda.

\section{FATORES PSICOLÓGICOS QUE INFLUENCIAM NA QUEIXA DE FALTA DE MEMÓRIA}

Os fatores psicológicos são o foco central quando se pesquisa sobre as dificuldades de memória. Idosos que apresentam queixas de dificuldades de memória, podem de fato indicar uma real dificuldade cognitiva, entretanto, estudos sugerem que estas queixas estejam mais associadas a depressão, estresse, ansiedade, autoestima, qualidade de vida, autoeficácia e metamemória (PAULO \& YASSUDA, 2009). Um estudo realizado com amostra de 67 idosos, apontou que a frequência de esquecimentos relatadas, esteve mais associada a sintomas de ansiedade do que de sintomas depressivos. Os resultados também apontaram que quanto maior a frequência de esquecimentos, maiores os sintomas de ansiedade. Além disto, os resultados apontaram que a autopercepção subjetiva de perda de memória dos idosos pesquisados, estaria mais associada à depressão e a traços de personalidade que ao desempenho objetivo nos testes neurológicos realizados (PAULO \& YASSUDA, 2009). Um dos achados do estudo de Santos et al. (2012), realizado com amostra de 204 idosos participantes saudáveis, foi a associação entre a percepção de estresse e a queixa subjetiva de comprometimento de memória. Foi verificado que quanto maior o nível de estresse informado pelos idosos, maior era a queixa de comprometimento de memória. Entretanto, é possível que a percepção de perda do funcionamento da memória constitui-se como um fator estressor, mediante o medo de vir a desenvolver demência. Entretanto, os autores identificaram que os idosos com maior queixa de comprometimento de memória apresentaram mais sintomas de depressão e pior autoestima do que os idosos sem queixa.

Quanto às queixas subjetivas de dificuldade de memória e traços de personalidade, os autores apontam que os dados apresentados são inconclusivos, uma vez que sujeitos com um traço afetivo mais depressivo e que referem sentimentos negativos em relação às suas 
habilidades com maior frequência, são mais propensos a relatarem pior funcionamento da memória em atividades cotidianas. Outros pesquisadores corroboram esta ideia, inclusive, é considerada a hipótese de que o desempenho ou as queixas subjetivas de dificuldade de memória também podem ser negativamente influenciada por atitudes e crenças a respeito de sua capacidade de memorização.

Apesar da limitação de literatura sobre este tema, Yassuda et al. (2006) realizaram uma pesquisa com 33 adultos e 27 idosos e corroboraram achados internacionais a respeito da autoeficácia, que, para os idosos foi mais baixa do que para os adultos. Entretanto, quando esta diferença é calculada de maneira geral, torna-se estatisticamente insignificante. Neste estudo, o fator ansiedade, esteve presente na amostra da população idosa. Demais autores, como Pergher, Stein \& Wainer (2004) e Melo et al. (2013) corroboram a ideia de que eventos estressores prejudicam não apenas a cognição de maneira geral, mas também a qualidade de vida.

\section{FATORES SOCIOECONÔMICOS E CLÍNICOS ASSOCIADOS À FALTA DE MEMÓRIA EM IDOSOS}

As funções cognitivas refletem amplamente as circunstâncias ambientais e a classe social que o idoso está inserido. Souza \& Wescher (2013) especificam que os níveis de escolaridade, habilidades intelectuais e qualidade de vida exercem significativa influência na memória dos idosos. No que se refere à qualidade de vida, idosos que mantinham preservadas a autonomia, independência e participação em atividades, apresentaram melhores resultados nas funções cognitivas, bem como é considerado que os idosos que mantiverem a prática regular de atividade física poderiam minimizar os declínios da memória declarativa (CORDEIRO et al., 2014).

Charchat-Fihman et al. (2005) realizaram uma revisão de estudos sobre processos fisiológicos do envelhecimento normal. Os autores identificaram que as variáveis demográficas, clínicas, ambientais e genéticas influenciam o desempenho dos idosos nos testes neuropsicológicos.

As comorbidades físicas estão associadas aos idosos e, assim, deixa-os cada vez mais expostos a polifarmacoterapia, definida pelo uso de cinco ou mais medicamentos por indivíduo (ROBERTI, 2017). Achados da revisão bibliográfica desta autora apontam que os medicamentos da classe dos betabloqueadores prejudicam a memória e que o uso de benzodiazepínicos são potencialmente nocivos à saúde, bem como alguns antidepressivos 
tricíclicos que possuem efeitos colaterais e geram efeitos anticolinérgicos, o que pode agravar quadros de confusão mental em idosos.

\section{PROGRAMA ZEN SÊNIOR COMO RECURSO TERAPÊUTICO}

A partir de uma perspectiva multicausal dos fenômenos relacionados aos diferentes graus de agravos à saúde, o estresse pode ser visto como um construto capaz de ampliar o grau de compreensão sobre a influência de processos sociais e psicológicos nos processos de adoecimento e de controle da saúde. No Brasil, segundo publicações do Instituto de Psiquiatria do HC (USP), aproximadamente $12 \%$ da população sofre de ansiedade, o que representa quase 24 milhões de brasileiros com ansiedade patológica (TAMAYO et al., 2004).

A interação social, sobretudo com pessoas da mesma geração, possibilita ao idoso construir novos laços de relação, favorecendo o bem-estar físico, psicológico e social. Desta forma, o programa Zen Sênior enfatiza em suas práticas a importância de adotar hábitos de vida saudáveis (físicos e emocionais) para a promoção de saúde mental.

Este programa tem como público-alvo adultos a partir de 55 anos que tenham queixas de estresse, ansiedade e dificuldades de memória e/ou atenção. Em um grupo de 8 participantes, são realizados 8 encontros semanais com duração de 60 minutos, facilitado por uma psicóloga em todos os encontros, e conta com o auxílio de uma nutricionista e um médico da Atenção Personalizada à Saúde - APS.

A Psicologia contribui com o acompanhamento dos beneficiários, orientando sobre o processo terapêutico, e técnicas para gerenciamento de estresse e ansiedade com foco em Atenção Plena (Mindfulness). As práticas são realizadas focadas no momento presente por meio de atenção na respiração, nos sons do ambiente e a utilização constante dos cinco sentidos para observar o contexto (visão, audição, tato, olfato e paladar). São realizados momentos de autocompaixão - regaste da história de vida com olhar de carinho e acolhimento - aceitação e diversas outras técnicas voltadas para ressignificar crenças limitadoras em relação a si mesmo e sua condição cognitiva e assim trabalhar sentimentos como autoestima e autoeficácia (HIRAYAMA et.al., 2014).

Na perspectiva da psicologia, a prática meditativa (mindfulness) tem sido definida como um treino sistemático da regulação atencional e emocional e possibilita o desenvolvimento de habilidades para mudar o foco de pensamentos e sentimentos, atuando essencialmente no controle da ansiedade, estresse e nas crenças relacionadas a autoeficácia e metamemória (CARPENA \& MENEZES, 2018). 
A nutrição participa de um encontro e contribui com atividades educativas voltadas a sensibilização do processo de alimentação, resgatando o controle e o prazer pela alimentação sem culpa, desconstruindo estigmas e crenças limitadoras, valorizando o momento da refeição e conscientizando sobre hábitos alimentares voltados à Atenção Plena na alimentação - Mindful eating (SILVA \& MARTINS, 2017).

O médico da Atenção Primária à Saúde realiza a aplicação do questionário sobre o perfil saúde e doença, identificação dos fatores de risco e acompanhamento da evolução dos pacientes durante o programa, visando a integralidade do cuidado (MENDES, 2010). Como instrumento de medida de desempenho e evolução do participante do programa, é utilizado a escala MAASMindful Attention Awareness Scale (FIGURA 1), composta por quinze itens com o objetivo de avaliar a atenção voltada para a consciência do momento presente e avalia, exclusivamente, o aspecto atencional da atenção plena (BARROS, 2015).

\section{Escala de Atenção e Consciência Plenas (MAAS)}

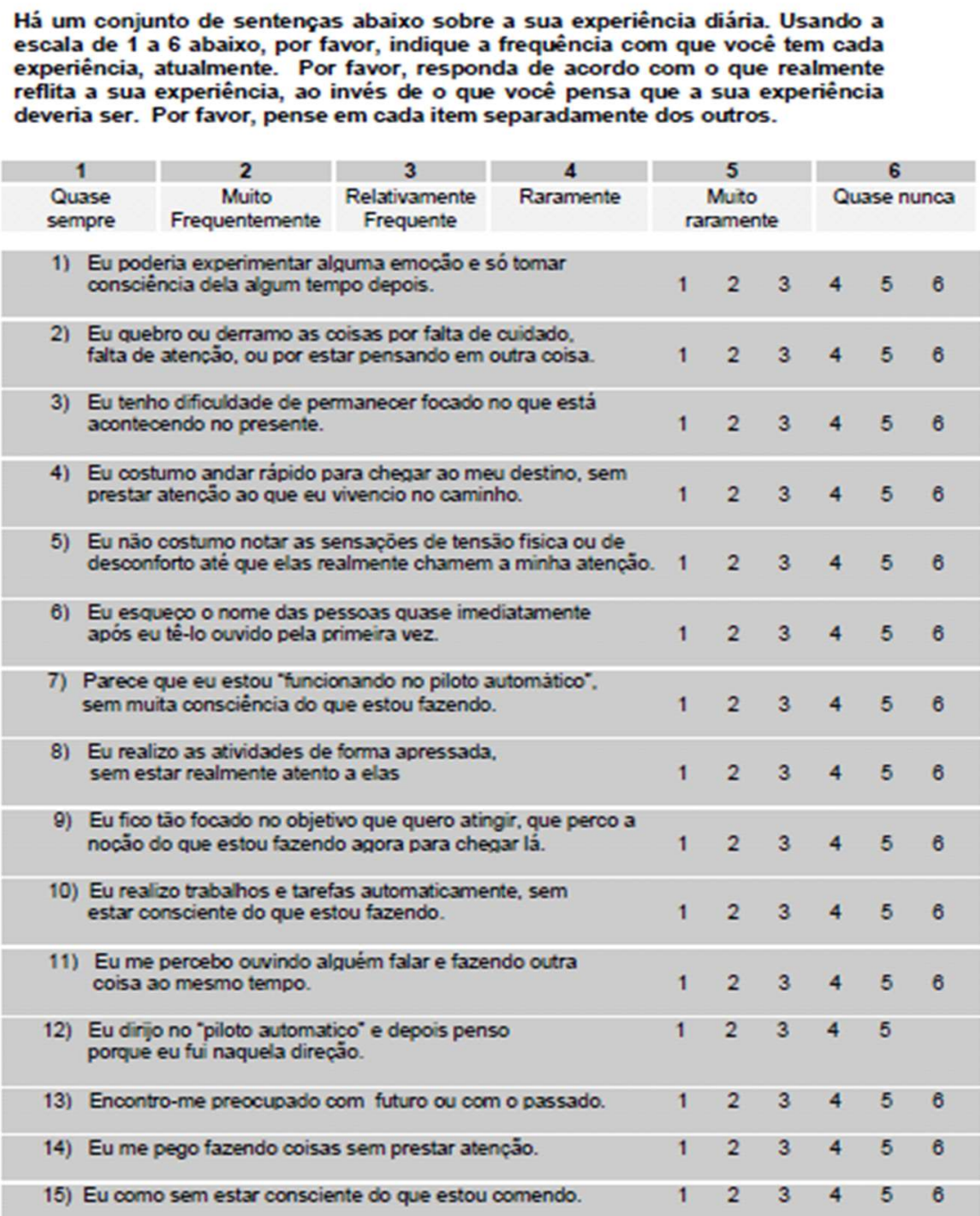




\section{CONSIDERAÇÕES FINAIS}

Os achados deste estudo apontaram fatores importantes que influenciam nas queixas de dificuldade de memória em idosos na esfera psicológica, socioeconômica e clínica/funcional. Os fatores ansiedade, estresse, traços de personalidade, declínio funcional e envelhecimento, foram indicados pelos estudos com forte associação às queixas de dificuldades de memória. Em contrapartida, sentimentos de autoeficácia, metamemória, qualidade de vida e prática de exercícios físicos foram apontados como fatores "fortalecedores" ao desempenho da memória em idosos. Quanto à depressão e ao uso de benzodiazepínicos, embora cotidianamente estejam associados às queixas, as pesquisas atuais não apontaram dados significativos para respaldar os efeitos destes nas queixas de memória.

O programa Zen Sênior surge como um recurso terapêutico a adultos e idosos que desejam melhorar sua qualidade de vida, nas esferas emocionais e cognitivas, com práticas meditativas, de autoconhecimento e de gerenciamento de estresse e ansiedade. Uma das limitações deste estudo refere-se a falta de materiais de avaliações pós-grupo para mensurar quantitativamente as melhoras dos beneficiários, uma vez que até o momento os relatos de melhora foram subjetivos, porém expressivos, pois o programa teve seu início em janeiro de 2019.

Este artigo procurou fazer uma breve descrição dos fatores associados à queixa de dificuldade de memória em idosos, onde enfaticamente os fatores psicológicos ficaram evidentes. Observa-se a necessidade da implementação de programas interdisciplinares, voltados a este tema para adultos e idosos no âmbito preventivo e de promoção de saúde.

\section{REFERÊNCIAS}

AMODEO, M. T.; NETTO, T. M.; FONSECA, R. P. Desenvolvimento de programas de estimulação cognitiva para adultos idosos: modalidades da Literatura e da Neuropsicologia. Letras de Hoje. Porto Alegre, v. 45, n. 3, p. 54-64, 2010.

APA - AMERICAN PSYCHIATRIC ASSOCIATION. Manual Diagnóstico e Estatístico de Transtornos Mentais. Trad. Cláudia Dornelles. $4^{\mathrm{a}}$ edição revisada, Porto Alegre, Artmed, 2002.

BARROS, V. V.; KOZASA, E. H.; SOUZA, I. C. W.; RONZANI, T. M. Evidências de Validade da Versão Brasileira da Escala de Atenção e Consciência Plena (MAAS). Psicologia: Reflexão e Crítica, v. 28, n. 1, p. 87-95, 2015.

BICCA, M. G.; ARGIMON, I. I. L. Habilidades cognitivas e uso de benzodiazepínicos em idosas institucionalizadas. Jornal Brasileiro de Psiquiatria, v. 57, n. 2, p. 133-138, 2008. 
CARPENA, M. X.; MENEZES, C. B. Efeito da Meditação Focada no Estresse e Mindfulness Disposicional em Universitários. Psicologia Teoria e Pesquisa. v. 34, 2018.

CARVALHO, F. C. R.; NERI, A. L.; YASSUDA, M. S. Treino de memória episódica com ênfase em categorização para idosos sem demência e depressão. Psicologia: Reflexão e Crítica, v. 23, n. 2, p. 317-323, 2010.

CHARCHATT-FITMAN, H. et al, C Declínio da capacidade cognitiva durante o envelhecimento, Revista Brasileira de Psiquiatria, v. 27, n. 12, p. 79-82, 2005.

CORDEIRO, J. et al., Efeitos da atividade física na memória declarativa, capacidade funcional e qualidade de vida em idosos, Rev. Brasileira de Geriatria e Gerontologia, v. 17, n. 3, p. 541-552, 2014.

ROBERTI, F. Medicamentos em idosos que causam prejuízo à memória. In: XVIII JORNADA DE EXTENSÃO, 18, 2018, Ijuí. Anais...Ijuí: Salão do Conhecimento, 2017.

HIRAYAMA, M. S. et al. A percepção de comportamentos relacionados à atenção plena e a versão brasileira do Freiburg Mindfulness Inventory. Ciênc. saúde coletiva., v. 19, n. 9, p. 3899-3914, 2014.

HUF, G.; LOPES, C. S.; ROZENFELD, S. O uso prolongado de benzodiazepínicos em mulheres de um centro de convivência para idosos. Cadernos de Saúde Pública, v. 16, n. 2, p. 351-362, 2000.

FERREIRA, H. G.; BARHAN, E. J. O envolvimento de idosos em atividades prazerosas: revisão da literatura sobre instrumentos de aferição. Revista Brasileira de Geriatria e Gerontologia, v. 14, n. 3, p. 579-590, 2011.

MELO, R. L. P. et al. O Efeito do Estresse na Qualidade de Vida de Idosos: O Papel Moderador do Sentido de Vida. Psicologia: Reflexão e Crítica, v. 26, n. 2, p. 222-230, 2013.

MENDES, E. V. As redes de atenção à saúde. Ciência \& saúde coletiva, v. 15, n. 2, p. 2297$2305,2010$.

MULLER, M. R.; GUIMARÃES, S. S. Impacto dos transtornos do sono sobre o funcionamento diário e a qualidade de vida. Estudos de Psicologia, v. 24, n. 4, p. 519-528, 2007.

PAULO, D. L. V.; YASSUDA, M. S. Queixas de memória em idosos e sua relação com escolaridade, desempenho cognitivo e sintomas de depressão e ansiedade. Revista Psiquiatria Clínica, v. 37, n. 1, p. 23-26, 2009.

PERGHER, G. K.; STEIN, L. M., WAINER, R. Estudos sobre a memória na depressão: achados e implicações para a terapia cognitiva. Revista Psiquiatria Clínica, v. 31, n. 2, p. 8290, 2004.

SANTOS. A. T., et al. Queixa subjetiva de comprometimento da memória em idosos saudáveis: influência de sintomas depressivos, percepção de estresse e autoestima, Revista da escola de Enfermagem da USP, v. 46, 2012. 
SILVA, B. F.; MARTINS, E. S. Mindfuleating na nutrição comportamental. In: Anais IX Simpac. Revista Científica Univiçosa - n. 1, Viçosa/MG, 2017.

SOUZA, A. A. F.; WECHSLER, S. M. Inteligência e criatividade na maturidade e velhice. Psicologia: Reflexão e Critica, v. 26, n. 4, p. 643-653, 2013.

TAMAYO, A. (Org.) Cultura e saúde nas Organizações. Porto Alegre: Artmed Editora, 2004. 255p.

YASSUDA, M. S.; LASCA, V. B.; NERI, A. L. Meta-memória e auto-eficácia: Um estudo de validação de instrumentos de pesquisa sobre memória e envelhecimento. Psicologia: Reflexão e Crítica, v. 18, n. 1, p. 78-90, 2005.

YASSUDA, M. S. et al. Treino de memória em idoso saudável. Psicologia: Reflexão e Crítica, v. 19, n. 3, p. 470-481, 2006. 